\title{
Cranial Capacity Estimates for Olduvai Hominid 7
}

\author{
MILFORD H. WOLPOFF \\ Department of Anthropology, University of Michigan, Ann Arbor, Michigan \\ 48109
}

KEY WORDS Cranial capacity, Australopithecine, OH 7, Homo habilis

\begin{abstract}
Estimation of cranial capacity for Olduvai Hominid (OH) 7 is determined from external parietal dimensions using multiple regressions calculated from an australopithecine grade sample. Capacity estimates for OH 7 (580-600 cc) are much lower than usually claimed. While differences in reconstruction may account for the varying estimates, a regression based only on undistorted and unreconstructed values, as well as a direct comparison of dimensions with other Homo habilis specimens, supports the smaller capacity determination.
\end{abstract}

It would be an understatement to say that times have changed since the discovery and initial interpretation (Leakey, 1961) of the juvenile hominid specimen from Olduvai gorge, $\mathrm{OH}$ 7. Still, some issues concerning the comparative morphology of the specimen have yet to be settled. Of these, the question of the cranial capacity of the vault represented by the two partial parietals has probably received the greatest attention.

Holloway's most recent discussion of the OH 7 reconstruction (1980) is an important contribution as well as a useful clarification of some longstanding issues. He has helped clarify the question of whether there was substantial twisting in the reconstructed fit between the reconstructed parietals. However, other issues remain. It is clear, as Holloway states, that the condition of the parietals, the amount of bone preserved, and the accuracy of the reconstruction renders them anything but useless as a basis for volume estimations. Indeed, I know of no worker who has claimed that they were unsuitable for this purpose. However, suitability is one thing, and the appropriateness of the estimation is quite another. It is in this area that much of the controversy has been focused.

The fact is that the parietals were crushed flat and have been reconstructed, and that before Holloway's most recent paper the volumetric estimates based on this reconstruction were determined from a biparietal partial endocast procedure that has never been adequately tested for accuracy (for details of the reconstruction and the biparietal partial endocast method, see Tobias, 1971). Holloway's most recent approach, using multiple regression techniques based on measurements taken from other endocasts appears to be more straightforward. Yet, his results still depend on the accuracy of the reconstruction, as well as on the sample used to generate the regression. With all due respect for Holloway's experience and appreciation of his substantial efforts, I wish to take issue with both of these points.

\section{PROBLEMS IN THE RECONSTRUCTION}

I believe that there are some problems in the reconstruction of the parietals that lead to uncertainties in their alignment relative to each other, and in their paracoronal curvature. The correct determination of sagittal length and biparietal breadth for the reconstructed parietal pair depends on both the reconstruction of the coronal curvature and on the parietal alignment in the coronal plane. Moreover, there is an obvious difference in paracoronal curvature between the left and right bones. These problems are further complicated by the lack of any bone-to-bone contact along the sagittal suture. Thus, it should be remembered that the reconstruction of the parietal pair that has been used in Holloway's various analyses is based on a positioning of the two parietals relative to each other that is determined by a set contact of bone with plaster

Received November 14, 1980; accepted July 24, 1981 
along the length of the sagittal suture. In the central portion of this suture, the contact is between plaster and plaster.

\section{Coronal curvature}

Each reconstructed parietal is made up of a number of bone fragments, clearly delineated by cracks that appear on both the internal and external surfaces. The positions of these fragments and their contacts are completely unambiguous. However, I question some of the angles created between the individual fragments at these contacts. For instance, on the left bone, the paracoronal curvature of the bone surface seems continuous over the inferior and central portions of the parietal wall. However, at its most superior aspect, the two large fragments bearing the preserved (anterior) portion of the sagittal suture seem to be set at an abrupt angle to the rest of the parietal. The sagittal suture is unduly elevated above the surface defined by the immediately contiguous bone fragments. As a result, if this parietal is aligned by positioning the inferiorcentral portion vertically, the bone surface just below the sagittal suture-bearing fragments is oriented almost exactly horizontally, but the two fragments with suture angle markedly upwards toward the midline (accentuating what I believe is their incorrect orientation). This actually produces a concave groove along a line roughly paralleling the sagittal suture but between this suture and the temporal line. On the other hand, if these suturebearing fragments are used to orient the remainder of the parietal by positioning their bone surface horizontally (as is required by the bone-to-plaster contact of the present reconstruction), then the central and inferior portion of the bone has a very marked angulation to the vertical and the maximum biparietal breadth, which is located along the inferior border, is significantly expanded. I believe the present reconstruction is incorrect, both because of the angulation of the most superior bone fragments (described above) and because of the angulation of the parietal sides to the vertical, which is unmatched in other early hominid sub-adults such as Taung (as suggested by the endocast form), SK 54 (Ron Clarke reconstruction), Omo 338y-6, and Modjokerto.

The set of the most posterior (sagittal suture-bearing) portion of the right side, relative to the plaster reconstruction of the remaining (anterior) sagittal suture-bearing region, shows that this side also contributed to what I regard as the inflated biparietal breadth.
Here, there are two bone fragments that reach the midline at the lamboidal region. When oriented along the midline as defined by the plaster sagittal suture reconstruction, the most posterior aspect of the actual suture is positioned more superiorly than the most anterior aspect. The problem in this case is in the plaster portion, but this is what defines the contact with the other side and the orientation of the combined parietals.

The effect of these problems on the reconstruction of the combined parietals can be outlined by the contrasting biparietal breadth estimates that probably represent a maximum and minimum. Holloway uses the existing reconstruction of the two parietals, mixing plaster and bone, in which all sagittal suture on one side is aligned by its contact with plaster on the other. The maximum biparietal breadth for this reconstruction is approximately 115 $\mathrm{mm}$. I have used the criteria of approximate verticality or only slight slope of the parietal sides, and alignment of the superior portion of the bones compensating for the angled fragments described above. In six determinations the maximum value for biparietal breadth I estimated is $102 \mathrm{~mm}$. The poorly oriented superior left parietal fragment discussed above would seem to result in a keeled sagittal region at this breadth. However, realignment of this fragment to conform to the curvature of the rest of the parietal would result in a flattened superior parietal region.

\section{Sagittal alignment}

The other problem to be noted in the present "set" reconstruction of the parietals is the alignment along the sagittal suture (i.e., along the plaster-to-bone contact). This is not a question of whether the sides are symmetric, or whether the parietals have been twisted relative to each other (the propositions tested by Holloway, 1980), but rather whether one has been translated relative to the other along the sagittal plane.

The exact positioning of the parietals along the sagittal plane is not simple or completely obvious, since there are only a limited number of corresponding points preserved on the two bones. The posterior inferior corner and the most inferior portion of the lambdoidal suture is preserved on both sides, as are many details of the meningeal artery impressions. When the two fragments are aligned using the comparable landmarks on the posterior inferior corner, the "plaster bregma" on the right side is at least $7 \mathrm{~mm}$ anterior to the real bregma 
preserved on the left. This indicates that there is a problem, and that the bregma-lambda dimensions used by Holloway may be too long.

In an attempt to ascertain the correct alignment along the sagittal plane, I used three preserved points to orient the bones relative to each other. These were the mastoid angle, the posterior edge of the bevel for the temporal squama, and the base of the middle meningeal artery impression. The parietals were reoriented on three separate occasions, and in all three cases the bregma-lambda distance was less than along the existing plaster and bone suture on the reconstruction. Interestingly, in his attempt to determine whether there is distortion of this reconstruction, Holloway concludes, "the most conspicuous region of distortion is on the left side, in the posterior region, roughly $2 \mathrm{~cm}$ from asterion (p. 271)." This should alert one to a problem since the region is one of the few that can be directly compared from side to side on the preserved bones.

In sum, my reconstruction of sagittal length is somewhat less than that used by Holloway. Tobias (1971) gives a bregma-lambda arc length of $105 \mathrm{~mm}$ for the reconstruction. My estimate, based on the average of the three attempts described above, is $95 \mathrm{~mm}$. Which, if either, of these estimates is more likely correct can never clearly be settled. However, the fact that my estimate is less than the $101 \mathrm{~mm}$ bregma-lambda arc value measured on ER 1470 and the value used by Holloway is greater, although every other parietal arc dimension for $\mathrm{OH} 7$ is less than the ER 1470 value (see Table 4), provides added support for the smaller determination.

\section{CRANIAL CAPACITY ESTIMATION}

The differences in reconstruction discussed have no effect on the affinities of $\mathrm{OH} 7$. Moreover, differences in cranial capacity estimation based on these different reconstructed values similarly do not effect an evaluation of these affinities, in my view, since both relatively large-brained (ER 1470) and smallbrained (ER 1813) specimens have been attributed to the taxon for which $\mathrm{OH} 7$ is a type specimen. The issues now are somewhat different than they were when $\mathrm{OH} 7$ was the only Homo habilis specimen with a vault and the estimation of its capacity was important with regard to whether the taxon was valid. At this time most workers (including myself) regard the specimen as representing Homo habilis, an australopithecine grade species on the clade that is ancestral to Homo erectus and contem- porary with a different australopithecine grade species, Australopithecus boisei. Moreover, that the range of cranial capacity variation in Homo habilis includes large-brained specimens is clearly indicated by males such as ER 1470.

Thus the estimated capacity of this particular specimen is not critical with regard to the species characteristics of Homo habilis. If it has any importance beyond the accurate assessment of the $\mathrm{OH} 7$ characteristics, it is probably in reference to how cranial capacity can be estimated for incomplete specimens. It is here that I differ from Holloway.

As Holloway (1980) correctly assumed (see footnote to Holloway's Table 6), the contention of a smaller capacity for $\mathrm{OH} 7$ was based on the external bony measurements. The two issues I am concerned with are whether external bony measurements of the parietal can provide an estimation of cranial capacity for less than complete fossil hominid specimens, and how such an estimation can best be determined. These issues are interdependent; the solution to the second is only relevant if the answer to the first is positive, but the answer to the first depends on the solution to the second!

\section{Multiple regression approach}

I attempted to estimate cranial capacity from linear dimensions using multiple regression techniques, just as Holloway did, although my efforts involved the external bony dimensions rather than the endocast dimensions. Because the possible distortions discussed above could affect point-to-point measurements, I have chosen parietal arcs covering continuous bone surface instead of chords, and have added to these the maximum breadths across the parietals, asterion, and the parietal mastoid angles. Six arc measurements and these three breadths were measured on the original specimen. The arcs were bregma-lambda, lambda-asterion, lambda-parietal mastoid angle, bregma-asterion, bregma-parietal mastoid angle, and bregma-krotaphion. [The parietal mastoid angle, as defined by Weidenreich (1943), is the angle on the temporoparietal suture located just over the mastoid, at or near the most posterior inferior extension of the bevel for the temporal squama; krotaphion is taken at the anterior inferior corner of the parietal where it meets the temporal squama and is not equivalent to pterion]. In the analysis that follows, all measurements were taken by me on the original specimens, assuring that 
the measuring points were defined the same way and thus that the measurements are fully comparable.

\section{Choice of sample}

My choice of the sample used to determine the multiple regression formula differs from Holloway's, who used varying base samples with different proportions of hominids and pongids (three pongid species, Homo sapiens, and an assemblage of fossil hominids spanning the last 3 million years). I disagree with this procedure because an estimation determined by a multiple regression is most accurately applied to the sample from which the regression was calculated (Pearson, 1926). In this case there are only three Homo habilis specimens with known capacities $(\mathrm{OH} 24$, ER 1470 , and 1813), all determined by Holloway (1978). ${ }^{1}$ It should be mentioned that Holloway expresses some uncertainty with respect to the $\mathrm{OH} 24$ capacity estimation of $590 \mathrm{cc}$. Yet, the point he questions is whether this estimate may be too large (p. 383), especially in view of some of its vault dimensions. Using the maximum vault capacity reported for it in my study acts to weigh the case in favor of a large estimte for $\mathrm{OH} 7$, since if the capacity estimate for $\mathrm{OH} 24$ really is too large, its use in a regression would tend to cause overestimates of cranial capacity from vault dimensions.

\section{Five approaches}

The first approach in estimating a capacity for $\mathrm{OH} 7$ was to use the two complete Homo habilis specimens (ER 1813 and ER 1470) to generate nine different linear equations for cranial capacity as a function of each measurement. Each of these equations was then used to estimate the $\mathrm{OH} 7$ cranial capacity, based on the measurement in question. The maximum and minimum determinations were omitted, and the average for the remaining seven capacity estimates is $578 \mathrm{cc}$ (the standard deviation is 43.5 ).

The second approach was to include $\mathrm{OH} 24$ in the Homo habilis sample. This resulted in a smaller number of usable measurements since all of the arcs could not be unambiguously determined for $\mathrm{OH} 24$ (for instance, no arcs spanning areas reconstructed in plaster were used). The cranial breadths used for this specimen were all reconstructed symmetrically from the undistorted right side of the base. Since there were more measurements (six) than specimens (three), I first determined which single measurement gave the lowest mean error ${ }^{2}$ for the sample of three in a single variable linear regression to predict cranial capacity. In this case the lambda-asterion arc gives a mean error of only $28.3 \mathrm{~mm}$. This arc was then combined with each of the remaining five independent variables resulting in five two-variable multiple regressions, each of which exactly predicted the three cranial capacities used in its determination.

These five regressions were then used to estimate the $\mathrm{OH} 7$ cranial capacity. Omitting the maximum and minimum estimates results in a sample of three estimates, averaging 574 cc (the standard deviation is 14.7). This estimate is probably the most accurate that can be determined for $\mathrm{OH} 7$ from the Homo habilis sample alone.

Of course, three specimens are a very small sample. Although this is the most appropriate sample to use, there can be little confidence in the results taken by themselves. A larger sample is clearly desirable. However, I believe that the choice of a larger sample should be constrained by the limitations of what a regression does and where it is most validly applied. Consequently, I contend that the next best sample to use is determined by closeness of relationship, as dictated by grade and clade. Because Homo habilis is an australopithecine grade species, other australopithecines are the best sample from which to determine a predictive regression. However, of the recognized australopithecine species, at least two clades are represented; Australopithecus africanus very likely is the species of the same clade that is closest in grade to Homo habilis, and I believe it is from this taxon that additional specimens for a wider based regression should be drawn. This provides five more specimens with cranial capacities determined by Holloway (1978): STS 5, STS 71, TM 1511 (STS 60),

'Holloway's reconstructions are the most recent of numerous at tempts for some of these specimens, and are widely used in the field. Whether or not they are correct for these more complete specimens (than $\mathrm{OH}$ 7), the fact that all of the comments addressed to this problem have proceeded from this assumption makes it necessary to accept the assumption of accuracy here. Otherwise, the discussion of technique becomes confused with the question of whether the base sample is accurate. In this way, at least the starting point for each procedure is the same.

${ }^{2}$ The mean error was determined as the average of the absolute differences between the actual and calculated cranial capacities. This statistic was used instead of the standard error for the adjusted standard error) so as not to unduly weigh outlying deviations in de termining the regression accuracy (the standard errors depend on the square of the difference). The coefficient of determination and the multiple correlation were not used because they provide a measure of the correlation between the actual and estimated values, and not a measure of how closely these actually approximate each other. 
MLD 37, and the juvenile specimen Omo $338 y-6$ [which, contra Rak and Howell (1978), I do not believe either represents a juvenile of $A$. boisei or differs from $A$. africanus in any significant way]. ${ }^{3}$

Using only the nine measurements that could be determined for $\mathrm{OH} 7^{4}$ (described above), a multiple regression for predicting cranial capacity was determined from these eight specimens. A stepwise approach was again employed, with each step chosen to minimize the mean error of the predicted values compared with the known values. The best fitting three-variable formula is given in Table $1 .{ }^{5}$ The mean error for this formula is 6.4 and the standard error is 11.7. This formula predicts a capacity of about $600 \mathrm{cc}$ for $\mathrm{OH} 7$. The best fitting four-variable formula (Table 2) has a mean error of 1.5 and a standard error of 4.6. The $\mathrm{OH} 7$ prediction is about $605 \mathrm{cc}$.

One could object to this third approach, arguing that some of the measurements used represent judgements made in reconstruction. In particular, the bregma-lambda arc depends on the fit of the parietals over the sagittal suture and, as mentioned above, there is no point at which bone is present on both sides of the suture. The maximum parietal breadth is influenced by the angulation of the parietals relative to each other, and this in turn is affected by the reconstruction of vault curvature near

$T A B L E$ 1. Best fitting three-variable regression for the Homo habilis and Australopithecus africanus combined sample. ${ }^{1}$ Regression: Cranial capacity $=3.2 *$ (biparietal breadth $)+$
$9.3 *$ (bregma-lambda arc) $+4.6 *$ (lambda-asterion arc) $-921.9 \mathrm{~mm}$

For the sample used in the regression

\begin{tabular}{lcc} 
& \multicolumn{2}{c}{ Cranial capacity } \\
\cline { 2 - 3 } & Actual & Predicted \\
\hline OMO 338y-6 & 427 & 414 \\
STS 71 & 428 & 436 \\
MLD 37 & 435 & 432 \\
STS 5 & 485 & 489 \\
ER 1813 & 509 & 520 \\
OH 24 & 590 & 587 \\
ER 1470 & 770 & 767 \\
\hline
\end{tabular}

For $\mathrm{OH} 7$, cranial capacity $\cong 600 \mathrm{cc}$

Measurements are in $\mathrm{mm}$ and capacity in cc. In this table, as well as in Tables 2 and 3 , the equation coefficients and constan have been rounded to one decimal place. However, the actual capacity calculations were based on the unrounded values and thus will be slightly different from those based on the formulae presented. the sagittal suture, as discussed above. My estimates to these relations differ from Holloway's, and I have used my own determinations in these regression calculations.

This problem can be avoided if the controversial measurements are excluded from the regression attempts. Consequently, a fourth approach was employed in which only arc measurements between landmarks connected by continuous bone surface on $\mathrm{OH} 7$ were used to determine a multiple regression from the eight specimens of Australopithecus africanus and Homo habilis. In this analysis, all observable effects of distortion and differing reconstructions are removed since the reconstructed or questionable measurements are omitted. The measurements used are the last five in the list

${ }^{2} \mathrm{Rak}$ and Howell cite "the marked prominence of the inferior temporal and the superior nuchal lines and the coarseness of the planum nuchale in an individual so young (p. 346)" as the primary support for placing this individual in Australopithecus boisei. However, the fact is that the specimen's age is unknown (for instance, it may have been a late adolescent with all but $\mathrm{M}^{3}$ erupted), and in any event virtually nothing is known of the age-specific ontogeny of these features in any australopithecine group. A very well-developed masticatory apparatus and prominent lines of muscle attachment characterize some South African Austrolopithecus africanus crania such as STS 71, and the elongated vault as seen from above more closely resembles this species than it does the globular form of the Australopithecus botsei vault. Holloway's recent analysis of the endocast (1981) also supports the contention that this Omo juvenile probably is not Australopithecus boisei.

${ }^{4} \mathrm{As}$ in the case of $\mathrm{OH} 24$, only measurements that could be clearly defined and were not obviously altered by distortion were used for this comparative sample.

${ }^{5}$ In each case, the formula was only determined for those specimens in the comparative sample that had the full measurement set (these are specificially listed in Tables 1-4). Thus, no attempt was made to approximate missing data.

TABLE 2. Best fitting four-variable regression for the Homo habilis and Australopithecus africanus combined sample.'

Regression: Cranial capacity $=3.5 *$ (biparietal breadth) + $12.3 *$ (bregma-lambda arc) $+4.9 *$ (lambda-asterion arc) $-2.8 *$ (bregma-parietal mastoid angle arc) $-949.3 \mathrm{~mm}$. For the sample used in the regression

\begin{tabular}{lcc} 
& \multicolumn{2}{c}{ Cranial capacity } \\
\cline { 2 - 3 } & Actual & Predicted \\
\hline OMO 338y-6 & 427 & 428 \\
STS 71 & 428 & 429 \\
MLD 37 & 435 & 431 \\
STS 5 & 485 & 486 \\
ER 1813 & 509 & 511 \\
ER 1470 & 770 & 769 \\
\hline
\end{tabular}

For $\mathrm{OH} 7$, cranial capacity $\cong 605 \mathrm{cc}$

Measurements are in $\mathrm{mm}$ and capacity in ce. 
above. The best fitting four-variable regression (Table 3) resulted in a cranial capacity estimate of about $570 \mathrm{cc}$ for $\mathrm{OH} 7$, with a mean error of 10.5 and a standard error of 31.4

While this is not the most accurate of the determinations in terms of the mean error, the fact that it is based only on measurements that were neither distorted nor affected by reconstruction in $\mathrm{OH} 7$, and presumably would be agreed upon by Holloway and me, suggests that it might be the most realistic estimate. Moreover, the fact that this estimate lies only slightly below the two multiple regression estimates discussed above indicates that the narrower reconstruction may be the more accurate one since a broader vault would have resulted in even larger capacity estimates (see below).

Finally, the expectation of a cranial capacity estimate for $\mathrm{OH} 7$ between the values for $\mathrm{OH}$ 24 and ER 1813, and much smaller than the ER 1470 capacity, is supported by a comparision of these five undistorted arc measurements for the Homo habilis specimens (Table 4).

\section{DISCUSSION}

In sum, five different regression attempts and a straightforward comparison provide surprisingly similar results for the estimation of $\mathrm{OH} 7$ cranial capacity, clustering within about $25 \mathrm{cc}$ (three within $8 \mathrm{cc}$. A value of approximately $580-600 \mathrm{cc}$ would seem to be a reasonable estimate, if not an overestimate, based on these determinations, since the mean for the five estimates is about $585 \mathrm{cc}$ (with a standard deviation of 16.0). If this exceeds the not "significantly in excess of $500 \mathrm{cc}$ " I have suggested before (Wolpoff and Brace, 1975), it falls even further from the $700-750 \mathrm{ml}$ range given as the "most probable estimate" by Holloway (1980).

It is reasonable to ask why these results differ considerably from the estimates made using the biparietal partial endocast method and from the estimates made from Holloway's regressions. Indeed, why can Holloway report that the $\mathrm{OH} 7$ parietals fit over endocasts with even larger volume? I believe that the answers to these questions lie in two independent factors, the inflated size of the present reconstruction and the choice of regression sample Holloway used in his estimation calculations.

\section{Partial endocasts}

The biparietal endocast method is itself a regression-type approach (although not actually a regression) since it is based on the average ratio of biparietal endocast volume to total en. docast volume. The sample used to determine
TABLE 3. Best fitting four-variable regression for the Homo habilis and Australopithecus africanus combined sample, using only arc measures not reflecting reconstruction or obvious distortion in $\mathrm{OH} 7^{1}$

Regression: Cranial capacity $=12.6 *$ (lambda-asterion arc) $+13.4 *$ (bregma-asterion arc) $-8.1 *$ (bregma-krotaphion arc) $-6.9 *$ (lambda-parietal mastoid angle arc) $-663.9 \mathrm{~mm}$

For the sample used in the regression

\begin{tabular}{lcc} 
& \multicolumn{2}{c}{ Cranial capacity } \\
\cline { 2 - 3 } OMO 338y-6 & Actual & Predicted \\
STS 71 & 427 & 420 \\
MLD 37 & 428 & 442 \\
STS 5 & 435 & 446 \\
ER 1813 & 485 & 461 \\
ER 1470 & 509 & 515 \\
\hline
\end{tabular}

For $\mathrm{OH} 7$, cranial capacity $\cong 570 \mathrm{cc}$

'Measurements are in $\mathrm{mm}$ and cranial in $\mathrm{cc}$.

TABLE 4. The Homo habilis sample compared for cranial capacity and for those parietal arc measures that do not reflect reconstruction or distortion in $\mathrm{OH} 7$

\begin{tabular}{lcccc}
\hline & ER & OH & OH & ER \\
& 1813 & 7 & 24 & 1470 \\
\hline $\begin{array}{l}\text { Cranial capacity } \\
\text { Parietal arcs }\end{array}$ & 509 & & $590^{1}$ & 770 \\
Bregma-krotaphion & 76.5 & 80.0 & & 88.0 \\
Lambda-asterion & 64.5 & 67.0 & 72.0 & 78.0 \\
Lambda-PMA & 76.8 & 85.0 & 87.0 & 88.0 \\
Bregma-asterion & 113.0 & 121.0 & & 132.0 \\
Bregma-PMA & 106.5 & 109.0 & & 120.0 \\
\hline
\end{tabular}

'The initial capacity estimate (mean of eight determinations) was $560 \mathrm{cc}$ (Leakey et al., 1971). This higher, more recent, estimate is used so as not to bias the $O H 7$ estinate downwards.

the average ratio was chosen in a manner that is similar to that used to pick the regression sample for this study. Tobias (1971) reports that he used the average for four fossil hominids with reliable volumes: Taung, $\mathrm{OH} 5$, and one specimen of Homo erectus from Indonesia (Trinil 2) and one from China (CKT II). Thus, the sample was also drawn from the most closely related hominids, although covering a broader geographic range, a longer temporal span, and a greater taxonomic diversity than I have done here. Yet there is sufficient similarity in method to question why the results are different. In this case, I believe the answer lies fully in the dimensions of the different $\mathrm{OH} 7$ reconstructions.

Using the dimensions taken on the current $\mathrm{OH} 7$ reconstruction (i.e., those used by Holloway) would elevate the capacity estimates 
for those regressions that employ sagittal arc and biparietal breadth, bringing them more in line with the biparietal endocast determination. Yet, apart from the argument that there are morphological reasons to question the reconstruction that provides these larger dimensions (see above), the fact is that other regressions used here that do not use these dimensions would not be altered (for instance Table 3 , or the majority of the determinations used to calculate the average given under the discussion of the first and second approaches above).

Thus, if the larger dimensions were used, there would be two different capacity estimate clusterings. I believe that this provides supplementary evidence supporting the contention that the sagittal and biparietal dimensions of the current reconstruction are too large. Using the smaller dimensions that I have determined results in only a single clustering of capacity estimates. Moreover, the direct comparison of vault dimensions with the other more complete Homo habilis specimens (Table 4) also indicates that the smaller rather than the larger estimates (and consequently the dimensions from which they were determined) are more likely correct. The alternative interpretation is that parietal dimensions cannot be used to estimate cranial capacity because they give inconsistent results (depending on which set of dimensions are used). Should this be the case, it would follow that none of the estimates that any of us have attempted have validity.

\section{Different regression samples}

A second problem confounds the comparison with Holloway's most recent capacity estimation. This is the choice of regression sample. The only fully valid sample for generating a regression to estimate the $\mathrm{OH} 7$ capacity is the Homo habilis sample, and the determinations from this sample provide the same smaller regression estimates as the determinations from the larger sample I have chosen. Nonetheless, the fact that the sample is small, and the question of whether all of the specimens in it should be considered Homo habilis (for instance, some workers regard ER 1813 as an East African varient of Australopithecus africanus), could form the basis of valid criticism.

Yet, what are the alternatives? The larger sample I have chosen for comparison covers a wide timespan and a broad geographic range. Nonetheless, these are the specimens of similar grade that are most closely related to $\mathrm{OH}$ 7 , and because a regression is most validly ap- plied to the sample from which it was calculated, this surely must be the most appropriate regression set. The biparietal endocast values that have been accepted for almost two decades are based on a ratio average for an even smaller sample but covering a greater timespan, geographic range, and number of taxa (three taxa for four specimens), as well as mixing specimens of two different evolutionary grades. The sample that Holloway used involves an extraordinarily broad range of time, space, and taxonomic diversity, and while the resulting sample size is large, Holloway (1980) admits: "The multiple regression analyses give a wide range of values, depending on the samples chosen and their constituent members" [p. 273]. Unfortunately, this is as it should be. The question of which regression base sample is most appropriate cannot be resolved precisely because none of them really are appropriate. Pearson (1926) questioned whether regression equations calculated in one human population could be validly applied to another, and the biological diversity spanned by any of Holloway's samples is considerably larger.

One final aspect of this problem is the criticism that, even if the sample I have used is valid, a number of the capacities that have been used are questionable. For instance, Holloway's objections to his own $\mathrm{OH} 24$ estimate have been noted, and one could similarly question the STS 71 value because of the possibility of distortion in the posterior region. Similarly, Holloway and Tobias have arrived at very different values for MLD 37.

I believe this is a valid criticism, one which must be constantly borne in mind during any discussion of the $\mathrm{OH} 7$ capacity since every one of these other questionable australopithecine specimens is more complete than OH 7. I have used only Holloway's published capacities in my regressions for the sake of consistency and because he had enough confidence in them to publish them. Clearly, however, the $\mathrm{OH} 7$ estimate can be regarded with no more certainty than the estimates for the other specimens used to determine the regression. Since these other specimens also form the basis of comparison of Homo habilis with Australopithecus africanus, the problem here is much broader than the single issue of how large the $\mathrm{OH} 7$ vault might have been.

\section{SUMMARY}

In sum, the main differences in capacity estimates for $\mathrm{OH} 7$ involve the reconstruction of the parietal set and the choice of regression samples used to calculate cranial capacity 
from the dimensions of the reconstruction. I have argued that the most appropriate basis for such a regression is the hominid sample most closely related to $\mathrm{OH} 7$, and very similar results were obtained using two such samples (Homo habilis alone and a mixture of all those Homo habilis and Australopithecus africanus specimens with cranial capacities published by Holloway). I have further argued that, for a variety of reasons, the sagittal length and biparietal breadth of the current $\mathrm{OH} 7$ reconstruction are too large. Using the smaller dimensions I ascertained during my study of the original specimen, I have obtained regression estimates of the cranial capacity that are the same as the estimates based on regressions that do not use the dimensions in question. For these reasons, I contend that there is just cause for the claim that the $\mathrm{OH} 7$ capacity may have been considerably smaller than the minimum estimate of $700 \mathrm{cc}$ suggested by Holloway.

\section{ACKNOWLEDGMENTS}

I am very grateful to $M$. Leakey, R.E.F. Leakey, F.C. Howell, P.V. Tobias, A. Hughes, C.K. Brain, and E. Vrba for permission to examine the original specimens in their care and for the hospitality and encouragement I received during my visits. Research in paleoanthropology would be impossible without the active and continuing cooperation of these individ- uals, and the debt owed to them could never be overstated. I would also like to thank R.L. Holloway, who reviewed this paper several times and made substantive comments and suggestions that I believe have greatly improved it.

This work was supported by NSF grants BNS 76-82729 and GS-33035.

\section{LITERATURE CITED}

Holloway, RL (1978) Problems of brain endocast interpretation and African hominid evolution. In Jolly, CJ (ed): Early Hominids of Africa. London: Duckworth. pp. 379-402.

Holloway, RL (1980) The O. H. 7 (Olduvai Gorge, Tanzania) Hominid partial brain endocast revisited. Am. J. Phys. Anthropol., 53:267-274.

Holloway, RL (1981) The endocast of the Omo L338y-6 juvenile hominid: Gracile or robust Australopithecus? Am. J. Phys. Anthropol., 54:109-118.

Leakey, LSB (1961) New finds at Olduvai Gorge. Nature 189:649-650.

Leakey, MD, Clarke RJ, and Leakey ISB (1971) New hominid skull from Bed I. Olduvai Gorge, Tanzania. Nature, 232:308-312.

Pearson, K (1926) On the reconstruction of cranial capacity from external measurements. Man, 26:46-50.

Rak, Y, and Howell, FC (1978) Cranium of a juvenile Australopithecus boisei from the lower Omo Basin, Ethiopia. Am. J. Phys. Anthropol., 48:345-366.

Tobias, PV (1971) The Brain in Hominid Evolution. New York: Columbia University Press.

Weidenrich, F (1943) The skull of Sinanthropus pekinensis; A comparative study on a primitive hominid skull. Palaeontologia Sinica D, No. 10.

Wolpoff, MH, and Brace, CL (1975) Allometry and early hominids. Science, 189:61-63. 